\title{
Ácidos graxos trans em produtos alimentícios brasileiros: uma revisão sobre aspectos relacionados à saúde e à rotulagem nutricional
}

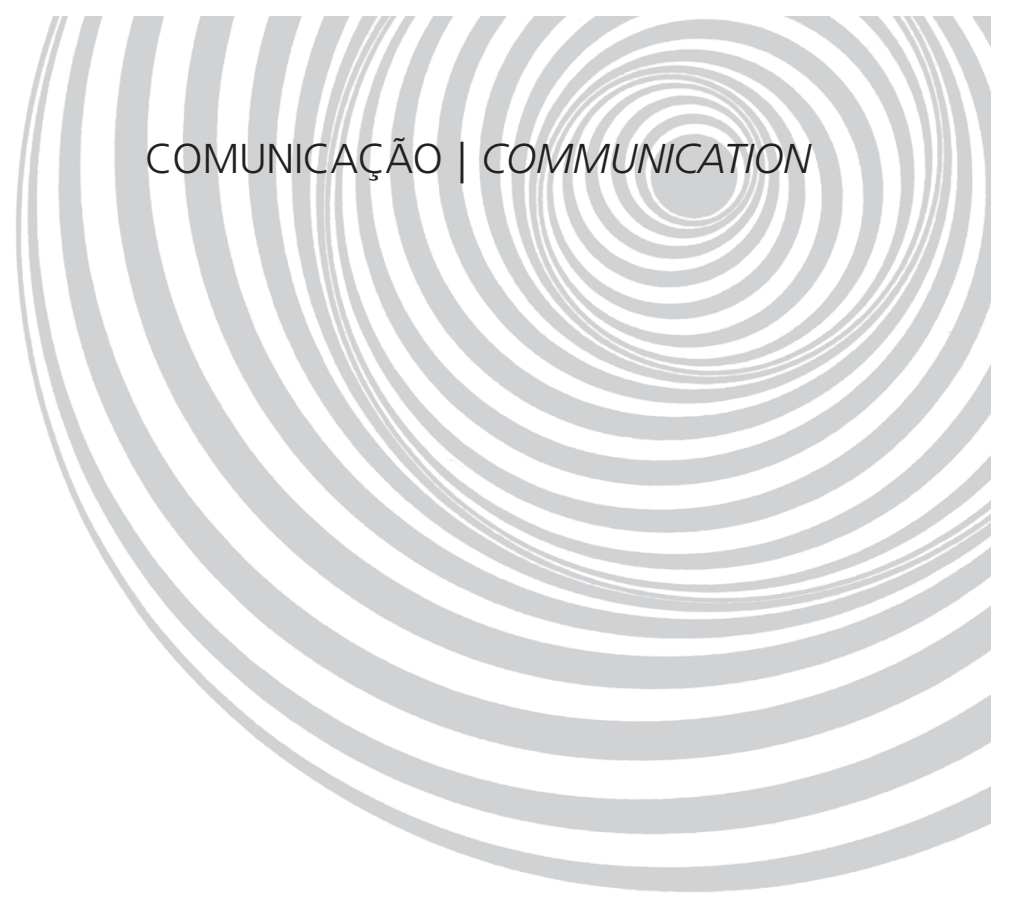

\author{
Trans fatty acids in Brazilian food products: \\ a review of aspects related to health \\ and nutrition labeling
}

Vanessa Martins HISSANAGA ${ }^{1}$

Rossana Pacheco da Costa PROENÇA ${ }^{1}$

Jane Mara BLOCK²

RE S U M O

Nos últimos anos, vários estudos apontam a relação positiva entre ácidos graxos trans e desenvolvimento de doenças cardiovasculares, materno-infantis, inflamatórias e de câncer. A Organização Mundial de Saúde alertou para a necessidade da diminuição do consumo desses ácidos graxos, o que culminou com a recomendação de sua eliminação em 2004. A rotulagem é uma medida que auxilia a população na escolha alimentar. Este artigo apresenta uma revisão sobre ácidos graxos trans, desde sua formação, efeitos para a saúde e as medidas atuais de controle da sua ingestão, enfatizando a rotulagem de alimentos. A busca de informações, relativa ao período de 1990 a 2012, foi realizada nos bancos de dados Scopus, PubMed, ScielO, Science Direct, Lilacs, bem como em sites oficiais nacionais e internacionais. Os unitermos utilizados em português e inglês foram: "ácidos graxos trans" e/ou "gordura hidrogenada", conjugados à "rotulagem" e/ou "regulação" e/ou "legislação". O controle dos ácidos graxos trans pode ser realizado pela diminuição de seu consumo por meio de medidas industriais, medidas individuais e coletivas, resultantes de um trabalho educativo, além de forças políticas. Em relação à rotulagem, mesmo existindo legislação brasileira que obrigue as indústrias a informarem a quantidade de ácidos graxos trans por porção em alimentos industrializados, observaram-se alguns questionamentos sobre como são disponibilizadas tais informações. Salienta-se que a efetiva diminuição dos ácidos graxos trans pode levar um tempo considerável, dada a adaptação cultural e tecnológica necessárias. Contudo, é uma medida importante, pois o resultado desse controle será a melhoria da saúde da população.

Termos de indexação: Ácidos graxos trans. Hidrogenação. Legislação. Rotulagem nutricional.

\footnotetext{
1 Universidade Federal de Santa Catarina, Núcleo de Pesquisa de Nutrição em Produção de Refeições. Campus Universitário, Trindade, 88040-900. Florianópolis, SC, Brasil. Correspondência para/Correspondence to: RPC PROENÇA. E-mail: rossana@mboxl.ufsc.br.

2 Universidade Federal de Santa Catarina, Programa de Pós-Graduação em Ciências de Alimentos. Florianópolis, SC, Brasil.
} 
518 | VM HISSANAGA et al.

\section{A B S T R A C T}

In recent years, several studies have indicated a positive relationship between trans fatty acids and cardiovascular disease, maternal and infant diseases, inflammatory diseases and cancer. The World Health Organization manifested the need of decreasing the consumption of these fatty acids, which culminated with the recommendation of their elimination in 2004. Labeling is a measure that helps the population make food choices. This article presents a literature review on trans fatty acids, their formation, health effects and the current measures to control their intake, emphasizing food labeling. The following databases were searched: Scopus, PubMed, SciELO, Science Direct and Lilacs. In addition, national and international government sites covering the period from 1990 to 2012 were consulted. The key words used in Portuguese and English were "trans fatty acids", and/or "hydrogenated fat", conjugated with "labeling", and/or "regulation", and/or "legislation". Manufacturing, individual and public measures stemming from governmental policies and education can help to reduce the consumption of trans fatty acids. Although Brazilian law requires companies to report the amount of trans fatty acids per serving on their product labels, the way this information is presented is questionable. The effective reduction of trans fatty acids in food products can take considerable time because of the necessary cultural and technological changes. It should be noted that any initiative regarding trans fatty acids is important since it will improve the general population's health.

Indexing terms: Trans fatty acids. Hydrogenation. Legislation. Nutritional labeling.

\section{N T R O D U ÇÃ O}

O consumo de ácidos graxos trans pela população vem aumentando desde 1920, quando teve início a produção industrial de gorduras vegetais'. A média de consumo estimada de ácidos graxos trans em países desenvolvidos é de 7-8g/dia por pessoa ${ }^{2}$. Larqué et al. ${ }^{3}$ reportaram valores na Europa entre 0,1g/dia e 5,5g/dia. No Japão, Semma ${ }^{4}$ estimou em 1,56g/dia.

Nesse contexto, Scheeder ${ }^{1}$ destaca que estudos têm ampliado o conhecimento sobre os efeitos negativos do consumo dos ácidos graxos trans para a saúde. As doenças mais associadas ao consumo desse ácido graxo são as doenças cardiovasculares e as crônicas degenerativas ${ }^{5-12}$; além disso, seu consumo influencia, do mesmo modo, o crescimento intrauterino, a obesidade e a doença inflamatóriå.

Diante dessa realidade, como resultado do aumento da disponibilização dos ácidos graxos trans e dos estudos que os associam a consequências negativas para a saúde, a Organização Mundial da Saúde (OMS) lançou, em 2004, a Estratégia Global para Promoção da Alimentação Saudável, Atividade Física e Saúde, estabelecendo a eliminação do consumo dos ácidos graxos trans como meta ${ }^{13}$.
Uma das medidas que podem auxiliar a população a realizar escolhas alimentares mais saudáveis em relação aos ácidos graxos trans é a rotulagem de alimentos. No Brasil, a Resolução da Diretoria Colegiada (RDC) no 360, de 23 de dezembro de 2003, tornou obrigatória a declaração do conteúdo de ácidos graxos trans por porção nos rótulos de todos os produtos alimentícios. No entanto, a resolução estabelece que possa ser considerado e divulgado como "zero trans" todo alimento que apresentar teor de ácido graxo trans menor ou igual a 0,2g/porção ${ }^{14}$.

Este texto objetiva, portanto, apresentar uma revisão bibliográfica sobre os ácidos graxos trans de origem industrial, seu consumo, consequências para a saúde e as possibilidades de controle da ingestão, discutindo uma possível lacuna na legislação brasileira para rotulagem nutricional.

\section{MÉ TOD OS}

A busca de informações foi realizada mediante revisão não sistemática de artigos apresentados nas bases de dados Scopus, Science, PubMed, SciELO e Lilacs, bem como em sites de organismos oficiais nacionais e internacionais, relativas ao período de 1990 a 2012. Os unitermos utilizados em português e inglês foram: "ácidos 
graxos trans, trans fatty acids", e/ou "gordura hidrogenada, hydrogenated fat", conjugadas à "rotulagem, labeling", e/ou "regulação, regulation", e/ou "legislação, legislation".

Durante a análise das publicações, agruparam-se as informações pertinentes às experiências de controle de disponibilização e/ou consumo de ácidos graxos trans, com foco na rotulagem de alimentos. A pesquisa não se pautou na preocupação numérica de garantir a representatividade proporcional dos achados para análise quantitativa, centrando-se no quanto as informações eram adequadas ao tema tratado. Dessa forma, artigos semelhantes foram excluídos e livros foram consultados.

\section{Formação dos ácidos graxos trans}

O consumo de ácidos graxos trans sempre fez parte da alimentação humana, pois eles estão presentes em gorduras de animais ruminantes, como resultado da bio-hidrogenação na microbiota do rúmen ${ }^{15,2}$. Estima-se que de $2 \%$ a $8 \%$ dos ácidos graxos trans da dieta sejam provenientes dessa fonte, dependendo do percentual individual de consumo desses produtos ${ }^{3}$.

Já cerca de $90 \%$ dos ácidos graxos trans da dieta são provenientes de óleos vegetais poli-insaturados que passaram pelo processo industrial de hidrogenação ${ }^{1,16}$, descrita como um processo no qual se tem a adição catalítica de uma molécula de hidrogênio na dupla ligação entre moléculas de carbonos. Como resultado, ocorre redução do grau de insaturação, além de seu aumento no ponto de fusão, resultando em uma maior estabilidade oxidativa e funcionalidade das frações semissólidas produzidas, sendo por isso amplamente utilizados pelas indústrias de alimentos ${ }^{2}$.

Apesar de se tratar, em ambos os casos, de isomeria trans, ressalta-se que na bio-hidrogenação há a produção de Ácido Linoleico Conjugado (CLA). Esse isômero natural, diferentemente dos produtos hidrogenados, vem sendo associado a benefícios para a saúde, como, por exemplo, a melhora no metabolismo plasmático de lipoproteínas ${ }^{17}$.

Ácidos graxos trans também podem ser formados, em quantidades menores, por mecanismo induzido termicamente em operações de frituras e durante o processo industrial de refino, principalmente na etapa de desodorização de óleos vegetais ${ }^{18,19}$. Durante a realização de frituras, a formação de ácidos graxos trans está relacionada ao tempo de uso e à temperatura atingida pelos óleos vegetais ${ }^{20}$.

Sanibal \& Mancini Filho ${ }^{20}$ realizaram estudo de fritura de batatas congeladas com o uso de óleo de soja e gordura parcialmente hidrogenada durante cinquenta horas e com temperatura controlada a Média $(\mathrm{M})=180^{\circ} \mathrm{C}$, Desvio-Padrão $(\mathrm{DP})=5^{\circ} \mathrm{C}$. O óleo de soja, inicialmente com 2,1\% de ácidos graxos trans, possivelmente formados durante o refino, no fim desse período totalizou $17,1 \%$ de isômeros trans. Já a fritura na gordura parcialmente hidrogenada apresentou menor formação de isômeros trans, embora a quantidade inicial fosse bem maior - 28,9\% -, e a quantidade final, 33,9\%.

Estudo realizado por Sebedio et al..$^{19}$ determinou a formação de isômeros trans em óleos de soja e de amendoim utilizados em fritura de batatas sob diferentes temperaturas: $180^{\circ} \mathrm{C}, 200^{\circ} \mathrm{C}$ e $220^{\circ} \mathrm{C}$, durante trinta operações. Após as temperaturas atingirem $200^{\circ} \mathrm{C}$, isômeros trans foram formados, em especial acima de dez utilizações desses óleos.

\section{Utilização da gordura parcialmente hidrogenada no processo industrial de alimentos}

Em 1903, o cientista alemão Wilhem Normann (1870-1939) patenteou a hidrogenação; após seis anos, o primeiro projeto industrial de hidrogenação foi construído na Inglaterra. A partir daí, várias outras indústrias foram sendo instaladas pelo mundo'. 
Segundo Scheeder ${ }^{1}$, o produto que marca a entrada dos óleos hidrogenados no mercado é a gordura vegetal Crisco, em 1912. Okie ${ }^{16}$ destaca que as demandas desses produtos para o consumo intensificaram-se durante tempos de guerras, em virtude do baixo custo e da maior estabilidade oxidativa, que confere aos alimentos um maior período de validade.

No Brasil, a hidrogenação de óleos vegetais teve uma ascensão significativa a partir da década de 1950, quando os óleos vegetais processados rapidamente substituíram as gorduras animais². Além do uso doméstico de gordura vegetal culinária, margarinas e gorduras para frituras, diversos produtos alimentícios, como biscoitos, produtos de panificação e batatas chips, dentre outros, são ricos em ácidos graxos trans ${ }^{7}$.

Block \& Barrera-Arellano ${ }^{21}$ analisaram o total de ácidos graxos trans de 42 amostras de margarinas, cremes vegetais e gorduras hidrogenadas comercializadas no Brasil em 1994, mediante espectroscopia no infravermelho. Os teores de isômeros trans variaram de $12,3 \%$ a 38,1\% (margarinas), de $15,9 \%$ a $25,1 \%$ (cremes vegetais) e de $0 \%$ a $62,0 \%$ (gorduras hidrogenadas), indicando quantidades significativas dos isômeros trans nesses produtos. Contudo, salienta-se que o estudo foi realizado anteriormente à legislação obrigatória de rotulagem de ácidos graxos trans em alimentos industrializados, podendo ser o motivo pelo qual se encontraram porcentagens elevadas dos isômeros nessas amostras.

Chiara et al. ${ }^{15}$ determinaram, por cromatografia gasosa, os teores de ácidos graxos trans, saturados, monoinsaturados e poli-insaturados em batatas fritas, biscoitos e sorvetes de redes de fast-food do Rio de Janeiro no ano de 2003. Verificou-se que apenas as batatas tipo chips não apresentaram teores de isômeros trans em sua composição. O estudo concluiu que em $100 \mathrm{~g}$ de biscoitos e sorvetes a quantidade de ácidos graxos trans era superior aos valores aceitos na época como limite: em média $1 \%$ do valor energético total diário. Cabe ressaltar que o estudo foi publicado no mesmo ano da legislação obrigatória de rotulagem de ácidos graxos trans no Brasil, época em que se observa a publicação de número representativo de estudos que associam o consumo de ácidos graxos trans a efeitos deletérios para a saúde humana.

Por sua vez, trabalho conduzido por Martin et al. ${ }^{7}$, realizado após o lançamento da legislação obrigatória de rotulagem de ácidos graxos trans no Brasil, objetivou determinar, por cromatografia gasosa, a composição de ácidos graxos de cinco marcas de biscoitos brasileiros do tipo cream cracker, e encontrou valores entre $12,2 \%$ e $31,2 \%$ de ácidos graxos trans do total lipídico, com a média de $20,1 \%$.

Na Áustria, Wagner et al.22 investigaram, por cromatografia gasosa, a quantidade de ácidos graxos trans do total lipídico de 400 amostras de produtos alimentícios e de alimentos de redes de fast-food. Aproximadamente um terço das análises apresentou teor de até $5,0 \%$ de ácidos graxos trans; contudo, 5,0\% apresentaram valores superiores a $20,0 \%$. Os produtos de redes de fast-food foram os que resultaram em maiores níveis, com média de 8,9\% de ácidos graxos trans.

Situação similar foi encontrada no estudo de Richter et al. ${ }^{23}$, que analisou o conteúdo de ácidos graxos trans por cromatografia gasosa de 119 produtos alimentícios adquiridos em Zurique, na Suíça. Os resultados apontaram que 40\% dos produtos apresentavam mais de $2 \%$ de ácidos graxos trans do total lipídico, e os produtos de confeitaria apresentaram uma média superior de $6 \%$ de ácidos graxos trans desse total.

Um documento publicado pela OMS ${ }^{24}$ aponta que a rotulagem de alimentos é incentivada não apenas no Brasil e demais países do Mercosul, mas em países da União Europeia, como Áustria e Suíça. Os estudos supracitados 7,15,22,23 destacam que, apesar da rotulagem, identificaram-se quantidades consideráveis de ácidos graxos trans nos produtos alimentícios analisados.

Um dos principais métodos utilizados em substituição à hidrogenação dos óleos vegetais é a interesterificação: processo que não promove a 
isomerização de duplas ligações dos ácidos graxos e não afeta o grau de saturação e permite que a modificação no comportamento de óleos e gorduras ofereça contribuições para o aumento e a otimização do seu uso 25 .

Entretanto, um artigo de revisão publicado em $2009^{26}$ destaca que a oferta desse substituto para a gordura parcialmente hidrogenada ainda é insuficiente para atender o mercado global. Além disso, pesquisas sugerem que gorduras interesterificadas podem causar malefícios para a saúde humana, como o aumento da glicemia e a diminuição do Lipoproteína de Alta Densidade-colesterol (HDL-c) $)^{27}$.

Nesse contexto, os autores L'Abbé et al..$^{28}$ e Zahn et al. ${ }^{29}$ ainda ressaltam que há dificuldades tecnológicas na substituição de produtos fontes de ácidos graxos trans em alimentos industrializados.

Os óleos de palma, de semente de palmiste e de coco são outros produtos que frequentemente substituem a gordura parcialmente hidrogenada em razão da consistência semissólida, além da facilidade do uso para produtos de padaria e frituras. Contudo, discute-se a eficácia dessa substituição, haja vista que a composição de seus ácidos graxos está por volta de $50 \%$ de saturação, sendo a gordura saturada frequentemente associada ao desenvolvimento de doenças cardiovasculares $^{30}$.

Os mesmos autores citados concluem que a tendência é a continuação dessa evolução de tecnologias, uma vez que se torna um desafio o encontro de um produto que atenda aos interesses comerciais das empresas e aos interesses dos consumidores, ou seja, aspectos relacionados a custo, sabor e saúde.

\section{Ácidos graxos trans e saúde}

Em 1961, alguns estudos já investigavam os efeitos da ingestão de gorduras hidrogenadas sobre os níveis de colesterol. Os resultados apontavam níveis mais elevados associados ao consu- mo de ácidos graxos saturados do que ao consumo de ácidos graxos trans. Dessa maneira, o uso de produtos ricos nesse ácido graxo continuou a fazer parte da alimentação da população ${ }^{31}$.

Mensink \& Katan ${ }^{32}$ despertaram a atenção de muitos pesquisadores ao mostrar que a ingestão elevada de ácidos graxos trans aumentava os níveis da Lipoproteína de Baixa Densidade-colesterol (LDL-C) de maneira similar aos ácidos graxos saturados. E, adicionalmente, reduziam os níveis da HDL-C, alterando significativamente a razão entre LDL-c e a HDL-c, utilizada como um importante indicador para as doenças cardiovasculares.

Nesse enfoque, estudos associam as alterações nas lipoproteínas sanguíneas em resposta ao consumo de ácidos graxos trans ao desenvolvimento de doenças cardiovasculares ${ }^{5-12}$. Expressando essa magnitude, Eckel et al. ${ }^{33}$ sugerem que um aumento no consumo de $2 \%$ de ácidos graxos trans pode ser responsável pela ascensão de 23\% na incidência das doenças coronarianas em adultos saudáveis.

Outro efeito do consumo dos ácidos graxos trans seria a interferência na saúde materno-infantil em razão de uma possível transferência dos isômeros consumidos pela gestante através da placenta. O resultado seria prejudicial ao feto, com sequelas no seu crescimento ${ }^{34,35}$.

Chiara et al..$^{34} \mathrm{e}$ Anderson et al. ${ }^{36}$ ressaltam que, após o nascimento do bebê, a presença de ácidos graxos trans na alimentação da mãe ainda pode comprometer a saúde materno-infantil pela possibilidade de transferência desses isômeros por meio do aleitamento.

Já o artigo publicado por Pisani et al. ${ }^{37}$ discute que o consumo de ácidos graxos trans na gestação e na lactação pode modificar tanto o perfil lipídico plasmático quanto alterar a expressão de adipocinas envolvidas com a resistência insulínica e doença cardiovascular dos descendentes. O estudo ainda salienta que esses efeitos deletérios estariam presentes mesmo após a retirada do fator causal.

Outros achados apontam relação positiva entre o consumo de ácidos graxos trans e male- 
fícios para a saúde, podendo ser citados: infertilidade feminina ${ }^{38}$, infertilidade masculina ${ }^{39}$, doença inflamatória ${ }^{40,41}$, depressão ${ }^{42}$, obesidade ${ }^{43}$ e câncer ${ }^{44,45}$.

Nesse contexto, o último compêndio de câncer, intitulado Food, Nutrition, Physical Activity, and the Prevention of Cancer: a global perspective (2007), sugeriu a diminuição do consumo de ácidos graxos trans como medida preventiva para a progressão da doença, uma vez que contribui positivamente para a melhoria e/ou manutenção do perfil nutricional do doente ${ }^{46}$.

\section{Consumo dos ácidos graxos trans}

Alguns trabalhos procuraram quantificar o consumo dos ácidos graxos trans por meio de análises da dieta. Valenzuela \& Morgado² apontam uma média de consumo em países desenvolvidos de sete a oito gramas por dia.

Allison et al. ${ }^{47}$ estimam que os norte-americanos consumam em média 5,3g/dia. Já para a população japonesa, Semma ${ }^{4}$ encontrou um consumo médio de 1,5g/dia. Embora os dados de todos os países não estejam completos, calculase que o consumo de ácidos graxos trans possa corresponder a 3,0\% (7,2g/dia) na Argentina; 2,0\% (4,5g/dia) no Chile; e 1,1\% (2,6g/dia) na Costa Rica ${ }^{48}$.

No Brasil, ainda não foi identificado estudo que estime o consumo dos ácidos graxos trans. Entretanto, Mondini \& Monteiro ${ }^{49}$ alertam que entre 1962 e 1988 o consumo de margarina no Brasil subiu de $0,4 \%$ para 2,5\% do total de calorias diárias. Também na Pesquisa de Orçamento Familiar (POF), realizada em 2002 e 2003, foi identificado um aumento de $16 \%$ no consumo de gorduras vegetais nos trinta anos anteriores ${ }^{50}$.

Salientam-se, além disso, dados da POF nos anos de 2008 e 2009, a partir dos quais, apesar da diminuição dos gastos com óleos e gorduras $(-1,1 \%)$, identificou-se o aumento da participação dos alimentos preparados $(+0,6 \%)$ e outros alimentos $(+1,1 \%)$, podendo sugerir ascensão de produtos industrializados, fontes potenciais de ácidos graxos trans ${ }^{51}$.

Estudo de Bertolino et al. ${ }^{52}$ analisou a influência da alteração do consumo de ácidos graxos trans nos níveis séricos de 328 nipo-brasileiros, de 40 a 79 anos, da cidade de Bauru (SP), entre 1993 e 2000. Mediante o uso de questionário de frequência alimentar, encontraram-se valores médios de ingestão de ácidos graxos trans (percentual de calorias totais) em: 5,1\% e 3,4\% para mulheres; $4,7 \%$ e 3,3\% para homens, em 1993 e 2000, respectivamente.

Em pesquisa realizada por Dias \& Gonçalves $^{53}$, que procurou identificar o consumo diário de ácidos graxos trans por intermédio de questionário de frequência alimentar de cem adultos e crianças de Duque de Caxias (RJ), concluiu-se que $39,7 \%$ e $41,4 \%$, respectivamente, consumiam pelo menos um alimento com alto teor de ácidos graxos trans ao dia.

Skeaff ${ }^{26}$ cita que, com exceção de alguns países, os bancos de dados de composição de alimentos não contêm informações sobre o conteúdo de ácidos graxos trans. Nesse aspecto, países como o Brasil podem ter dificuldade de estimar o consumo de ácidos graxos trans pela população, uma vez que os métodos de avaliação dietética podem ser caros e ter baixa precisão.

Contudo, salientam-se os estudos citados previamente, nos quais se encontraram teores consideráveis de ácidos graxos trans em produtos alimentícios e de redes de fast-food brasileiras e estrangeiras, ressaltando a crescente participação desses tipos de alimento na dieta dos brasileiros ${ }^{54,55}$.

Ainda destaca-se que frituras servidas em redes de fast-food são muitas vezes realizadas em gordura parcialmente hidrogenada. A rede McDonald's dos Estados Unidos da América anunciou em 2002 a troca do óleo utilizado para a batata frita por uma opção com menor teor de ácidos graxos trans. Contudo, a mesma empresa voltou a se pronunciar em 2003, alegando dificuldades com a redução do teor de ácidos graxos 
trans em suas preparações e não informando um novo prazo para essa diminuição ${ }^{56}$.

Estudo mais recente sobre a redução voluntária de ácidos graxos trans na América Latina e Caribe relata que de doze representantes de indústrias de alimentos que assinaram previamente uma declaração afirmando a intenção de eliminar os ácidos graxos trans de seus produtos, um ano depois apenas três forneceram detalhes completos das suas reformulações. Outros três forneceram apenas informações gerais e ainda outros três alegaram dificuldades para encontrar um substituto ideal da gordura parcialmente hidrogenada, o alto custo desses substitutos e a baixa aceitação sensorial dos consumidores dos produtos alimentícios após a substituição ${ }^{57}$.

Diante do exposto, pode-se inferir que a eliminação de ácidos graxos trans ainda é um desafio para a indústria de alimentos e para as redes de fast-food; sendo assim, o seu consumo ainda é uma realidade, especialmente para as pessoas que costumam consumir alimentos industrializados e/ou provenientes dessas redes.

\section{Diretrizes oficiais quanto ao consumo de ácidos graxos trans}

Organização Mundial da Saúde em 1995 sugeriu que a ingestão de ácidos graxos trans não ultrapassasse $1 \%$ do valor enérgico total diário, buscando promoção da saúde ${ }^{58}$. Em 2004, a mesma instituição lançou a Estratégia Global para Promoção da Alimentação Saudável, Atividade Física e Saúde, estabelecendo como uma de suas metas a eliminação do consumo de ácidos graxos trans produzidos industrialmente ${ }^{13}$.

Entretanto, no Brasil, apesar dos preceitos da mencionada estratégia da OMS, o Guia Alimentar da População Brasileira ${ }^{59}$ não aponta a eliminação de ácidos graxos trans como meta, mas sim restringe um limite correspondente a $1 \%$ do valor energético total diário, aproximadamente 2,2g/dia de uma dieta de 2000 calorias para um adulto saudável.
Assim, observa-se que, mesmo existindo um documento mais recente da $\mathrm{OMS}^{13}$, o Ministério da Saúde do Brasil aparentemente baseou-se na preconização de $1995^{58}$.

Organizações governamentais de alguns países como, por exemplo, Dinamarca, Canadá e Índia, além do estado de Nova York, nos Estados Unidos da América, atentos à recomendação da $\mathrm{OMS}^{13}$, estão promovendo iniciativas com o objetivo de diminuir a oferta dos ácidos graxos trans ${ }^{28}$. Outro exemplo de país articulado em torno da questão é a Escócia ${ }^{60}$.

Na Dinamarca, o primeiro país a trabalhar com esta temática, foi aprovada em 2004 uma legislação limitando a 2\% o conteúdo de ácidos graxos trans presente nas gorduras vegetais para consumo humano. Como esse limite é aplicado nas matérias-primas, na prática, o teor de ácidos graxos trans nos produtos alimentícios dinamarqueses fica bastante reduzido ${ }^{61}$.

Stender et al. ${ }^{62}$ avaliaram a exposição dos consumidores aos ácidos graxos trans por meio da análise de produtos alimentícios populares consumidos na Dinamarca e em outros 25 países durante o período de novembro de 2004 a fevereiro de 2006. Os resultados demonstraram uma menor quantidade dos isômeros trans nos produtos dinamarqueses. Assim, a legislação dinamarquesa pode ser considerada uma intervenção interessante por garantir alguma proteção à população, sem aparentemente causar efeitos sobre a disponibilidade e a qualidade dos produtos.

No mesmo foco de discussão, L'Abbé et al. ${ }^{28}$, em um artigo que objetivou analisar as iniciativas de diversos países na redução de ácidos graxos trans, apontaram as características comuns para as experiências bem-sucedidas: (1) a existência de especialistas nacionais envolvidos na questão e (2) o envolvimento da mídia no sentido de facilitar o acesso à informação para a população, que se torna mais bem preparada para cobrar do meio científico e da indústria medidas para a minimização do consumo de ácidos graxos trans. 
Nesse sentido, um grupo de trabalho nomeado Américas Livres de Gorduras Trans, envolvendo membros da academia e de órgãos oficiais, recomenda que a Organização Pan-Americana de Saúde/World Health Organization (OPASMHO) lidere as iniciativas para a eliminação progressiva dos ácidos graxos trans. Em abril de 2007, o grupo ainda propôs que o Comitê Executivo da OPAS avaliasse o relatório e o submetesse à consideração na Reunião de Conferência Sanitária Pan-Americana da OPAS em outubro daquele ano; entretanto, o assunto não entrou em discussão na ocasião ${ }^{48}$.

Em junho de 2008, o grupo de estudos da OPAS/OMS Américas Livres de Gordura Trans se reuniu e recomendou metas, tais como a continuação do trabalho dos estados membros para a harmonização continental das regulamentações de gorduras trans e a elaboração de programas para aumentar a conscientização dos consumidores ${ }^{63}$.

Outro grupo de trabalho da OMS, considerando evidências científicas, concluiu que os ácidos graxos trans produzidos industrialmente, como não estão naturalmente nos alimentos e não trazem benefícios para a saúde, podem ser considerados aditivos industriais e, como tal, restaurantes e fabricantes de alimentos devem evitá-los ${ }^{64}$.

\section{Rotulagem alimentar de ácidos graxos trans}

O Canadá, em 2003, foi o primeiro país a tornar obrigatória a rotulagem de ácidos graxos trans. Friesen \& Innis ${ }^{65}$ encontraram uma significativa redução de ácidos graxos trans no leite materno de mulheres canadenses após a regulamentação, comparando os resultados obtidos em 1998 com os obtidos no período entre 2004 e $2006^{65}$.

No Brasil, a Resolução RDC n 360 de 2003 tornou obrigatória a informação sobre a quantidade de ácidos graxos trans nos alimentos embalados a partir de julho de $2006^{14}$.
Assim, tornou-se obrigatória a declaração dos ácidos graxos trans em relação a uma porção de cada produto, determinada pela RDC $n^{\circ} 359^{66}$. Ainda, segundo a RDC $n^{\circ} 360^{14}$, podem ser considerados e divulgados como "zero trans" ou "não contêm trans" os alimentos que apresentarem teor de ácidos graxos trans menor ou igual a $0,2 \mathrm{~g} /$ porção, sendo o referido valor descrito como não significativo na resolução.

Observa-se, portanto, que a RDC $n^{\circ} 360^{14}$, lançada em dezembro de 2003, pode ter sido baseada na regulamentação da Food and Agriculture Organization/World Health Organization (FAO/ WHO) lançada no mesmo ano - a Diet Nutrition and Prevention of Chronic Diseases ${ }^{67}$ - e não na Estratégia Global para Promoção da Alimentação Saudável, Atividade Física e Saúde ${ }^{13}$, que recomenda a eliminação dos ácidos graxos trans produzidos industrialmente.

Nota-se que a indústria alimentícia vem destacando cada vez mais produtos com informações de fácil visualização, tal como, "zero trans". No entanto, cabe lembrar que as denominações referem-se a uma porção, e que se o consumo for superior a essa quantidade, pode ocorrer uma ingestão considerável desse isômero. Por exemplo, considerando-se a RDC $n^{\circ} 359^{66}$, uma porção de biscoito é equivalente a 2,5 unidades. Então, se em 2,5 unidades não se atinge 0,2 gramas de ácidos graxos trans, um pacote de biscoito pode anunciar que não apresenta o isômero. Contudo, se uma pessoa ingerir uma quantidade maior do que 2,5 unidades do biscoito em questão acumulará o consumo de ácidos graxos trans, podendo, eventualmente, alcançar o limite destacado pelo Guia Alimentar para a População Brasileira ${ }^{59}$, que é de 2,2 gramas/dia.

Salientam-se, também, as diversas maneiras de disponibilizar a informação sobre os ingredientes nos rótulos. Nesse sentido, um folder explicativo da Agência Nacional de Vigilância Sanitária (Anvisa) ${ }^{68}$ sobre a questão recomenda que "é importante também verificar a lista de ingredientes do alimento"(online), na qual é possível identificar a adição de gorduras hidrogenadas durante a fabricação. 
Pesquisa realizada em supermercado brasileiro, considerando rótulos de 2327 alimentos industrializados, encontrou que são utilizadas 14 denominações para designar a gordura trans na lista de ingredientes, desde o mais comum - "gordura vegetal hidrogenada" - até aqueles equivocados na denominação química - "óleo vegetal líquido e hidrogenado". A autora destaca que, quimicamente, somente pode-se chamar de gordura a substância que, na temperatura ambiente, for sólida ou semissólida, chamando-se de óleo apenas a substância líquida. Foram encontrados, também, nove denominações que deixam dúvida sobre o conteúdo de gordura trans, como "gordura vegetal" ou "margarina"69.

Assim, observando as recomendações, entende-se que possivelmente exista uma lacuna importante na legislação brasileira, já que a rotulagem e a apelação de ausência de ácidos graxos trans não podem ser consideradas completamente seguras, necessitando também a consideração da lista de ingredientes. Nesse mesmo contexto, cabe, igualmente, ressaltar que, quando a quantidade de ácidos graxos trans não alcança os 0,2g por porção, a empresa fica desobrigada a disponibilizar a quantidade desse ácido graxo, impossibilitando a análise nutricional quanto ao citado componente.

Ainda sobre a rotulagem de ácidos graxos trans, pesquisa de mercado realizada pelo Instituto Brasileiro de Defesa do Consumidor ${ }^{70}$ encontrou, em uma amostragem de 370 produtos, 37,6\% de inadequação à exigência da legislação, ou seja, ausência da informação regulamentada pela RDC $n^{\circ} 360^{14}$ quanto à presença ou não dos ácidos graxos trans.

Dias \& Gonçalves ${ }^{53}$, ao analisarem a rotulagem nutricional de 150 amostras de biscoitos, chocolates e sorvetes, além da informação nutricional de 49 amostras de hambúrguer, batata frita, milk-shake e sorvetes de redes de fast-food do Rio de Janeiro, constataram a ausência da apresentação do teor de ácidos graxos trans em $22,0 \%$ da amostra total, principalmente nos sorvetes $(38,7 \%)$ e biscoitos recheados $(27,1 \%)$.
Já a pesquisa de Gagliardi et al. ${ }^{71}$, que avaliou a composição nutricional de produtos alimentícios com alegação de zero gordura trans, encontrou a redução do lipídeo em alimentos como margarinas, biscoitos doces, biscoitos salgados, batatas fritas e lanches tipo hambúrguer de redes de fast-food, mas não sua ausência, como os consumidores acreditam ao comprar o produto. Assim, a ausência dos ácidos graxos trans nos produtos analisados pode ser considerada virtual, além de eles estarem, muitas vezes, sendo substituídos por ácidos graxos saturados, também positivamente associados a doenças cardiovasculares.

No trabalho publicado por Aued-Pimentel et al. ${ }^{72}$, que analisou por cromatografia gasosa 22 amostras de salgadinhos, batatas fritas, sorvetes, produtos de panificação, bebida láctea, creme vegetal e macarrão instantâneo, com a alegação nos rótulos de "0\% de gordura trans", os resultados indicaram desacordo em quatro amostras, ou seja, os valores obtidos variaram de $0,3 \mathrm{~g} /$ porção a 1,8g/porção, contrariando o limite de até $0,2 \mathrm{~g} /$ porção. Os autores ainda destacam que somente uma amostra apresentou claramente na lista de ingredientes descrita no rótulo a presença de gordura vegetal parcialmente hidrogenada.

Além dessas inadequações, estudo realizado por Ferreira et al. ${ }^{73}$ aponta que a RDC $n^{\circ} 360^{14}$ foi implementada sem que fosse acompanhada de campanhas de esclarecimento à população, que se ressente da falta do conhecimento do que venham a ser os ácidos graxos trans, podendo o termo ser erroneamente interpretado até como alimento transgênico.

Os rótulos devem ser um espaço para informação ao consumidor e, quando compreendidos, podem facilitar escolhas alimentares nutricionalmente mais criteriosas. Nessa direção, para que a rotulagem exerça sua função, as informações disponibilizadas devem ser fidedignas, legíveis e acessíveis ${ }^{74}$

Remig et al. ${ }^{75}$ concluem em um trabalho de revisão que a educação do consumidor é muito 
importante. Ressaltam que programas educacionais devem ser desenvolvidos visando à capacitação dos consumidores para identificarem a presença de gordura vegetal parcialmente hidrogenada na lista de ingredientes dos rótulos, bem como na estimativa mais acurada das porções dos produtos alimentícios a serem consumidos.

Uma medida que poderia auxiliar nessa estimativa éa recomendação do Codex Alimentarius, que aponta que a quantificação de nutrientes na rotulagem nutricional seja em $100 \mathrm{~g}$ ou $100 \mathrm{~mL}$, pois, assim, comparações diretas entre os produtos alimentícios são mais facilmente realizadas ${ }^{76}$.

Nesse contexto, fica evidente a necessidade do desenvolvimento de políticas públicas na área de educação e comunicação, visando auxiliar a população na apropriação das informações vinculadas pelos rótulos dos produtos alimentícios.

Pode-se então sugerir que, no Brasil, a preocupação com a presença de ácidos graxos trans nos produtos alimentícios é uma questão de saúde pública ainda não completamente equalizada nem pelos estudiosos, nem pela legislação, nem pela população, o que estimula a busca de estratégias de diversas esferas para a processual diminuição e a possível eliminação dos ácidos graxos trans na alimentação.

Sucintamente, L'abbé et al. ${ }^{28}$ apresentam as abordagens utilizadas em diversos países com o objetivo de diminuir o consumo dos ácidos graxos trans, destacando: (1) sensibilização da população sobre os efeitos do consumo de ácidos graxos trans, pautada em alegações de saúde; (2) rotulagem do teor de ácidos graxos trans obrigatória ou voluntária e (3) reformulação da indústria de alimentos, com a remoção dos ácidos graxos trans, voluntariamente ou sob forma de lei.

Recentemente, em nota técnica publicada sobre as ações do governo brasileiro no que concerne aos ácidos graxos trans ${ }^{77}$, foram elencadas ações prioritárias pelo Ministério da Saúde em parceria com a Anvisa.

Dentre essas ações, destaca-se a regulamentação da publicidade de alimentos com teores elevados de gordura saturada, gordura trans, açúcar e sódio. Essa regulamentação trouxe uma proposta baseada na obrigatoriedade da veiculação de alerta sobre o perigo do consumo excessivo de componentes não saudáveis nos alimentos. Representantes da indústria, da sociedade civil e das empresas de comunicação participaram da etapa final do texto da resolução publicada em 15 de junho de 2010 com a seguinte recomendação: "Alimento com quantidade elevada de gordura trans é aquele que possui em sua composição uma quantidade igual ou superior a $0,6 \mathrm{~g}$ para $100 \mathrm{~g}$ ou $100 \mathrm{~mL}$ na forma como está exposto à venda; o alimento que apresentar essa característica deve notificar a advertência: O nome/marca comercial do alimento contém muita gordura trans e, se consumida em grande quantidade, aumenta o risco de doenças do coração"78.

Salienta-se que a advertência pode ser caracterizada como uma iniciativa interessante para auxiliar os consumidores no controle do consumo dos ácidos graxos trans, bem como na divulgação da informação sobre os riscos à saúde decorrentes do consumo desse ácido graxo. Porém, discute-se o porquê dessa recomendação afirmar que alimento com quantidade elevada de ácido graxo trans é aquele que apresenta mais do que $0,6 \mathrm{~g}$ para $100 \mathrm{~g}$ do produto, citando a OMS ${ }^{13}$ que, desde 2004, aponta como meta a eliminação do consumo de ácidos graxos trans produzidos industrialmente. Nessa linha de pensamento, a preocupação é que o valor de $0,6 \mathrm{~g}$ de gordura trans para $100 \mathrm{~g}$ de produto alimentício tenha uma conotação de valor máximo permitido, dando a impressão de que qualquer valor abaixo dele possa representar um consumo seguro.

\section{CONSIDERAÇÕ ES FINAIS}

O tema deste artigo vem ao encontro da temática de estudos na área de Segurança Alimentar e Nutricional, ou seja, explora a rotulagem dos alimentos no Brasil, especificamente em relação aos ácidos graxos trans. 
Salienta-se que o consumo de ácidos graxos trans pela população cresceu paralelamente ao aumento do seu uso pelas indústrias de alimentos, havendo, ainda, a partir da década de 1990, inúmeros estudos publicados que apontam uma associação positiva com diversas doenças, principalmente as cardiovasculares.

O controle de ácidos graxos trans pode ser feito pela diminuição de seu consumo por meio de medidas industriais, com substituição de tecnologias. Tal controle pode abranger também medidas individuais e coletivas, resultantes de um trabalho educativo e também de forças políticas.

Um exemplo de país que pode ser seguido é o da Dinamarca, onde o teor de ácidos graxos trans deve ser inferior a $1 \mathrm{~g}$ por $100 \mathrm{~g}$ do total de óleo ou gordura do produto finalizado, o que na prática diminuiu a quase zero o teor do lipídeos nos alimentos industrializados.

Identificou-se uma lacuna importante na legislação brasileira no que tange à rotulagem de alimentos. Os questionamentos são, principalmente, sobre a maneira como são disponibilizadas a informação nutricional e a lista de ingredientes. Assim, sugere-se a necessidade de reformulação dessa legislação nos referidos aspectos, por exemplo, com a padronização do termo para se designar a gordura parcialmente hidrogenada, não podendo ser utilizada outras nomenclaturas, tal como gordura vegetal, capaz de facilitar dupla interpretação.

Outra sugestão que ajudaria na interpretação da rotulagem nutricional em relação aos ácidos graxos trans seria a informação por cada $100 \mathrm{~g}$ ou $100 \mathrm{~mL}$ do produto alimentício. Dessa for-ma, comparações entre produtos alimentícios de um mesmo grupo seriam realizadas mais facilmente, podendo posteriormente subsidiar escolhas nutricionalmente mais saudáveis.

Em relação a trabalhos educativos, sugere-se a extensa divulgação dos malefícios dos ácidos graxos trans em canais abertos de televisão e rádio e em escolas.

A efetiva diminuição do uso e do consumo de ácidos graxos trans pode levar um tempo considerável, dada a adaptação cultural e tecnológica que requer. Trata-se, contudo, de uma medida importante, considerando-se que o resultado desse controle será a melhoria da saúde da população, com consequente redução de gastos com saúde.

\section{COLABORADORES}

Todos os autores participaram da concepção e da redação do artigo.

\section{REFERÊ NCIAS}

1. Scheeder MRL. About the trans-(hi) story: how did trans fatty acids enter the human food chain. Am Oil Chem Soc. 2007; 18(2):133-5.

2. Valenzuela A, Morgado N. Trans fatty acid isomers in human health and in the food industry. Biol Res. 1999; 32(4):273-87. doi: 10.4067/S0716-976019 99000400007.

3. Larqué E, Salvador Z, Gil A. Dietary trans fatty acids in early life: a review. Early Hum Dev. 2001; 65 (Suppl): S31-S41.

4. Semma M. Trans fatty acids: properties, benefits and risks. J Health Sci. 2002; 48(1):7-13.

5. Oomen CM, Ocké MC, Feskens EJM, van Erp-Baart MAJ, Kok FJ, Kromhout D. Association between trans fatty acid intake and 10-year risk of cononary heart disease in the Zutphen Elderly Study: a prospective population-based study. Lancet. 2001; 357(9258):746-51.

6. Baylin A, Kabagambe EK, Ascherio A, Spiegelman D, Campos H. High 18:2 Trans Fatty acids in adipose tissue are associated with increase risk of nonfatal acute myocardial infarction in Costa Rican adults. J Nutr. 2002; 133(4):1186-91.

7. Martin CA, Matshushita M, Souza NE. Ácidos graxos trans: implicações nutricionais e fontes na dieta. Rev Nutr. 2004; 17(3):361-8. doi: 10.1590/S14 15-52732004000300009.

8. Costa AGV, Bressan J, Sabarense CM. Ácidos graxos trans: alimentos e efeitos na saúde. Arch Latinoam Nutr. 2006; 56(1):12-21.

9. Mozaffarian D, Clarke R. Quantitative effects on cardiovacular risk factors and coronary heart disease risk of replacing partially hydrogenated vegetable oils with other fats ond oils. Eur J Clin Nutr. 2009; 63:S22-S33. doi:10.1038/sj.ejcn.1602976. 
10. Mozaffarian D, Aro A, Willet WC. Health effects of trans-fatty acids : experimental and observational evidence. Eur J Clin Nutr. 2009; 63:S5-S21. doi: 10.1038/sj.ejcn.1602973.

11. Karbowska J, Kochan Z. Trans-fatty acids-effects on coronary heart disease. Pol Merkur Lekarski. 2011; 31(181):56-9.

12. Fournier N, Attia N, Rousseau-Ralliard D, Vedie B, Destaillats F, Grynberg A, et al. Deleterious impact of elaidic fatty acid on ABCA1-mediated cholesterol efflux from mouse and human macrophages. Biochim Biophys Acta. 2012; 1821(2):303-12. doi: 10.1016/j.bbr.2011.03.031.

13. World Health Organization. Who global strategy on diet, physical activity and health: list of all documents and publications. Fifty-sevenyh World Health Assembly [Internet]. A57/9, 17 abr. 2004a. [cited 2007 Aug 3] Available from: <http://apps. who.int/gb/ebwha/pdf_files/WHA57/A57_9en.pdf>.

14. Brasil. Ministério da Saúde. Agência Nacional de Vigilância Sanitária. Resolução RDC n³60, de 23 de dezembro de 2003. Aprova regulamento técnico sobre rotulagem nutricional de alimentos embalados, tornando obrigatória a rotulagem nutricional. Diário Oficial [da] República Federativa do Brasil. 200326 dez.

15. Chiara VL, Sichieri R, Carvalho TSF. Teores de ácidos graxos trans de alguns alimentos consumidos no Rio de Janeiro. Rev Nutr. 2003; 16(2):227-33. doi: 10.1590/S1415-52732003000200010.

16. Okie S. New York to trans fats: you're out! N Engl J Med. 2007; 356 (20): 2017-21.

17. Kritchevsky D, Tepper SA, Wright S, Czarnecki SK, Wilson TA, Nicolosi RJ. Conjugated linoleic acid isomer effects in atherosclerosis: growth and regression of lesions. Lipids. 2004; 39(7):611-6. doi: 10.1007/s11745-004-1273-8

18. Martin CA, Milinsk MC, Visentainer JV, Matsuschita M, de-Souza NE. Trans fatty acid-forming processes in foods: a review. An Acad Bras Ciênc. 2007; 79 (2): 343-50.

19. Sebedio JL, Catte M, Boudier MA, Prevost J, Grandgirald A. Formation of fatty acid geometrical isomers and of cyclic fatty acid monomers during the finish frying of frozen prefried potatoes. Food Research Intern. 1996; 29(2):109-16. doi:10.1016/ j.bbr.2011.03.031.

20. Sanibal EAA, Mancini Filho J. Alterações físicas, químicas e nutricionais de óleos submetidos ao processo de fritura. Food Ingredientes. 2002(18); 48-54.

21. Block JM, Barrera-Arelano D. Produtos hidrogenados no Brasil: isômeros trans, características físico-químicas e composição em ácidos graxos. Arch Latinoam Nutr. 1994; 44(4):281-5.

22. Wagner K-H, Plasser E, Proell C, Kanzler S. Comprehensive studies on the trans fatty acid content of Austrian foods: Convenience products, fast food and fats. Food Chemistry. 2008; 108(3): 1054-60. doi:10.1016/j.foodchem.2007.11.038.

23. Richter EK, Shawish KA, Scheeder MRL, Colombani PC. Trans fatty acid content of selected Swiss foods: the transswisspilot study. J Food Compos Anal. 2009; 22:479-84. doi:10.1016/j.jfca.2009.01.007.

24. World Health Organization. Nutrition labels and health claims: the global regulatory environment. Geneva: WHO; 2004.

25. Ribeiro APB, Moura JMLN, Grimaldi R, Gonçalves LAG. Interesterificação química: alternativa para obtenção de gorduras zero trans. Quím Nova Escol. 2007; 30(5):1295-300.

26. Skeaff CM. Feasibility of recommending certain replacement or alternative fats. Eur J Clin Nutrit. 2009; 63:S34-S49. doi: 10.1038/sj.ejcn.1602974.

27. Sundram K, Karupaiah T, Hayes KC. Stearic acidrich interesterified fat and trans-rich fat raise the LDL/HDL ratio and plasma glucose relative to palm olein in humans. Nutr Metab. 2007; 4(3):1-12. doi: 10.1186/1743-7075-4-3.

28. L'abbé MR, Stender S, Skeaff M, Ghafoorunissa, Tavella M. Approaches to removing trans fat from the food supply in industrialized and developing countries. Eur J Clin Nutr. 2009; 63:S50-S67. doi: 10.1038/ejcn.2009.14.

29. Zahn S, Pepke, F, Rohm H. Effect of inulin as a fat replacer on texture and sensory properties of muffins. Inter J Food Sci Technol. 2010; 45(12): 2531-7. doi: 10.1111/j.1365-2621.2010.02444.x.

30. Tarrago-Trani MT, Phillips KM, Lemar LE, Holden JM. New and existing oils and fats used in products with reduced trans-fatty acid contend. J Am Diet Assoc. 2006; 106(6):867-80.

31. Katan MB, Mensink RP. Trans fatty acids and their effect on lipoproteins in humans. Annu Rev Nutr. 1995; 15(5): 473-93. doi: 10.1146/annurev.nu.1 5.070195 .002353 .

32. Mensink RP, Katan MB. Effect of dietary trans fatty acids on high-density and low-density lipoprotein cholesterol levels in healthy subjects. N Engl J Med. 1990; 373(7):39-45.

33. Eckel RH, Borra S, Lichtenstein AH, Yin-Piazza SY. Understanding the complexity of trans fatty acid reduction in the American diet. Circulation. 2007; 115(16):2231-46. doi:10.1161/CIRCULATIONNA HA.106.181947.

34. Chiara VL, Silva R, Jorge R. Ácidos graxos trans: doenças cardiovasculares e saúde materno-infantil. 
Rev Nutr. 2002; 15(03):341-7. doi: 10.1590/\$141552732002000300010.

35. Eijsden MV, Hornstra G, Vander Wal MF, Vrijkotte TGM, Bonsel GJ. Maternal n-3, n-6, and trans fatty acid profile early in pregnancy and term birth weight: a prospective cohort study. Am J Clin Nutr. 2008; 87(4):887-95.

36. Anderson K, McDougald DM, Steiner-Asiedu M. Dietary trans fatty acid intake and maternal and infant adiposity. Eur J Clin Nutr. 2010; 64(11): 1308-15. doi:10.1038/ejcn.2010.166.

37. Pisani LP, Oller do Nascimento CM, Bueno AA, Biz C, Albuquerque KT, Ribeiro EB, et al. Hydrogenated fat diet intake during pregnancy and lactation modifies the PAl-1 gene expression in white adipose tissue of offspring in adult life. Lipids Health Dis. 2008; 7(13):1-10. doi:10.1186/1476-511X-7-13.

38. Chavarro JE, Rich-Ed-Edwards JW, Rosner BA, Willet W. Dietary fatty acid intakes and the risk of ovulatory infertility. Am J Clin Nutr. 2007; 85(1): 231-7.

39. Chavarro JE, Furtado J, Toth TL, Ford J, Keller M, Campos $\mathrm{H}$, et al. Trans-fatty acid levels in sperm are associated with sperm concentration among men from an infertility clinic. Fertil Steril. 2011; 95(5):1794-7. doi:10.1016/j.bbr.2011.03.031

40. Mozaffarian D, Pischon T, Hankinson SE, Rifai N, Joshipura K, Willet WC, et al. Dietary intake of trans fatty acids and systemic inflammation in women. Am J Clin Nutr. 2004; 79(4):606-12.

41. Bendsen NT, Stender S, Szecsi PB, Pedersen SB, Basu $\mathrm{S}$, Hellgreen $\mathrm{LI}$, et al. Effect of industrially produced trans fat on markers of systemic inflammation: evidence from a randomized trial in women. J Lipid Res. 2011; 52(10):1821-8. doi: 10.1194/Jr.M014 738.

42. Sánchez-Villegas A, Verberne L, de Irala J, RuízCanela M, Toledo E, Serra-Majem L, et al. Dietary fat intake and the risk of depression: the sun project. PLoS One. 2011; 6(1):e16268. doi:10.13 71/journal.pone.0016268.

43. Thompson AK, Minihane A-M, Williams CM. Trans fatty acids and weight gain. Inter J Obes. 2011; 35(3):315-24. doi:10.1038/ijo.2010.141 .

44. Chajés $V$, Thiébaut $A C$, Rotival $M$, Gauthier $E$, Maillard V, Boutron-Ruault M-C, et al. Association between serum trans-monounsaturated fatty acids and breast cancer risk in the E3N Study. Am J Epidemiol. 2008; 167(11):1312-20. doi: 10.1093/ aje/kwn069.

45. Vinikoor LC, Millikan RC, Satia JA, Schroeder JC, Martin CF, Ibrahim JG, et al. Trans-Fatty acid consumption and its association with distal colorectal cancer in the North Carolina Colon
Cancer Study II. Cancer Causes Control. 2010; 21(1):171-80. doi: 10.1007/s10552-009-9447-3.

46. World Cancer Research Fund. American Institute for Cancer Research. Food, nutrition, physical, and the prevention of cancer: a global perspective. Washington (DC): AICR, 2007.

47. Allison DB, Egan SK, Barraj LM, Caughaman C, Infante $\mathrm{M}$, Heimback JT. Estimated intakes of trans fatty and other fatty acids in the US population. J Am Diet Assoc. 1999; 99(2):166-74. doi: 10.1016/ j.bbr.2011.03.031.

48. Organização Pan-Americana da Saúde. Grupo de trabalho da OPAS/OMS Américas livres de gorduras trans: conclusões e recomendações de 26 e 27 de abril de 2007 [Internet]. Washington (DC): OPAS; 2007 [acesso 2007 ago 3]. Disponível em: <www. dpalsc.org>.

49. Mondini L, Monteiro CA. Mudanças no padrão de alimentação. In: e novos males da saúde no Brasil: a evolução do país e de suas doenças. São Paulo: Hucitec; 1995.

50. Instituto Brasileiro de Geografia e Estatística. Pesquisa de orçamentos familiares 2002-2003: análise da disponibilidade doiciliar e estado nutricional no Brasil. Rio de Janeiro: IBGE; 2004.

51. Instituto Brasileiro de Geografia e Estatística. Pesquisa de orçamentos familiares 2008-2009: despesas, rendimentos e condições de vida. Rio de Janeiro: IBGE; 2010.

52. Bertolino CN, Castro TG, Sartorelli DS, Ferreira MAC. Influência do consumo alimentar de ácidos graxos trans no perfil de lipídios séricos em nipobrasileiros de Bauru, São Paulo, Brasil. Cad Saúde Pública. 2006; 22(2):357-64. doi: 10.1590/S0102-31 $1 \times 2006000200013$.

53. Dias JR, Golçalves ECBA. Avaliação do consumo e análise da rotulagem nutricional de alimentos com alto teor de ácidos graxos trans. Ciênc Tecnol Aliment. 2009; 29(1):177-82.

54. Monteiro CA, Gomes FS, Cannon G. The Snack Attack. Am J Public Health. 2010; 100(6):975-81. doi: 10.2105/AJPH.2009.187666.

55. Monteiro CA, Levy RB, Claro RM, de Castro IRR, Cannon G. Increasing consumption of ultraprocessed foods and likely impact on human health: evidence from Brazil. Public Health Nutr. 2011; 14(1):5-13. doi: 10.1017/\$1368980010003241.

56. Katan MB. Regulation of trans fats: the gap, the Polder, and McDonalds French fries. Atheroscler Suppl. 2007; 7(2):63-6. doi: 10.1016/j.atherosclero sissup.2006.04.013.

57. Rojas R, Colón-Ramos U, Jacoby E, Mozaffarian, D. Voluntary reduction of trans-fatty acids in Latin America and the Caribbean: current situation. Am 
J Public Health. 2011; (2):126-29. doi: 10.1590/S1 020-49892011000200008.

58. World Health Organization. Nutrition. Science - Policy. WHO and FAO Joint Consultation: fats and oils in human nutrition. Nutr Rev. 1995; 53(7): 202-5

59. Brasil. Ministério da Saúde. Guia alimentar para a população brasileira: promovendo a alimentação saudável [Internet]. Brasília: MS, 2005 [acesso 2007 abr 10]. Disponível em: <http://www.saude.gov.br/ bvs>.

60. Simpson, R. Limit on trans fats (Scotland) Bill: Improving Scotland's diet and protecting public health; 2009 [cited 2010 Jul 4]. Available from: $<$ http://www.scottish.parliament.uk/S3_Members Bills/Draft\%20proposals/Finaltransfatsconsul tation.pdf $>$.

61. Leth $T$, Jensen $H G$, Mikkelsen $A E$, Bysted $A$. The effect of the regulation on trans fatty acid contend in Danish food. Atheroscler Suppl. 2006; 7(2):53-6. doi: 10.1016/j.bbr.2011.03.031.

62. Stender S, Dyerberg J, Bysted A, Leth T, Astrup A. A trans world journey. Atheroscler Suppl. 2006; 7(2):47-52. doi: 10.1016/j.bbr.2011.03.031.

63. Organização Pan-Americana da Saúde. Américas livres de gorduras trans: declaração do Rio de Janeiro de 08 e 09 de junho de 2008, Rio de Janeiro, RJ [acesso 2009 set 23]. Disponível em: <http://www.paho.org/Portuguese/AD/DPC/NC/ transfat-declaracao-rio.pdf>.

64. Uauy R, Aro A, Ghafoorunissa R, L'abbé ML, Mozaffarian D, Skeaff $M$, et al. Who Scientific Update on trans fatty acids: summary and conclusions. Eur J Clin Nutr. 2009; 63:S68-S75.doi: 10.1038/ejcn.2009.15.

65. Friesen $\mathrm{R}$, Innis SM. Trans fatty acids in human milk in Canada declined with the introduction of trans fat food labeling. J Nutr. 2006; 136(10):2558-61.

66. Brasil. Ministério da Saúde. Agência Nacional de Vigilância Sanitária. Resolução RDC n 359, de 23 de dezembro de 2003. Aprova regulamento técnico de porções de alimentos embalados para fins de rotulagem nutricional. Diário Oficial [da] República Federativa do Brasil. 200326 dez.

67. World Health Organization. Diet, nutrition and the prevention of chronic diseases. Geneva: WHO; 2003 [cited 2007 Aug 3]. Available from: <www. fao.org>.

68. Brasil. Ministério da Saúde. Folheto explicativo sobre rotulagem de gorduras trans [Internet]. Brasília: MS; 2006 [acesso 2007 nov 3]. Disponível em: <www.anvisa.gov.br/alimentos/gorduras_ trans.pdf>.

69. Silveira BM. Informação alimentar e nutricional da gordura trans em rótulos de produtos alimentícios industrializados [mestrado]. Florianópolis: Universidade Federal de Santa Catarina; 2011.

70. Instituto Brasileiro de Defesa do Consumidor. Bom para os olhos e o paladar, ruim para o coração. Idec [Internet]. 2006 [acesso 2007 nov 10]; 103. Disponível em: <www.idec.org.br/oq_idec.asp>.

71. Gagliardi ACM, Mancini Filho J, Santos RD. Perfil nutricional de alimentos com alegação de zero gordura trans. Rev Assoc Med Bras. 2009; 55(1): 50-3.

72. Aued-Pimentel S, Silva SA, Kus MMM, Caruso MSF, Zenebon O. Avaliação dos teores de gordura total, ácidos graxos saturados e trans em alimentos embalados com alegação "livre de gordura trans". Braz J Food Technol. 2009; VII BMCFB.

73. Ferreira $A B$, Lanfer-Marquez UM. Legislação brasileira referente à rotulagem nutricional de alimentos. Rev Nutr. 2007; 20(1):83-93. doi: 10.1590/S1415-52 732007000100009 .

74. Marins BR, Jacob SC, Peres F. Avaliação qualitativa do hábito de leitura e entendimento: recepção das informações de produtos alimentícios. Ciênc Tecnol Aliment. 2008; 28(3):579-85.

75. Remig V, Franklin B, Margolis S, Kostas G, Nece T, Street JC. Trans fats in America: a review of their use, consumption, health implications, and regulation. J Am Diet Assoc. 2010; 10(4):585-92. doi: 10.1016/j.bbr.2011.03.031.

76. World Health Organization. Understanding the Codex Alimentarius [Internet]. Roma: WHO; 2005 [cited 2010 Jul 4]. Available from:<ftp://ftp.fao.org/ codex/Publications/understanding/Understanding_ EN.pdf>.

77. Brasil. Ministério da Saúde. Ações do Governo Brasileiro sobre gordura trans, Rio de Janeiro [Internet]. 2008 [acesso 2010 jul 4]. Disponível em: <http://189.28.128.100/nutricao/docs/geral/ nota_imprensa_gorduras_trans.pdf $>$.

78. Brasil. Ministério da Saúde. Agência Nacional de Vigilância Sanitária. Resolução - RDC n²4, de 15 de junho de 2010. Dispõe sobre a oferta, propaganda, publicidade, informação e outras práticas correlatas cujo objetivo seja a divulgação e a promoção comercial de alimentos considerados com quantidades elevadas de açúcar, de gordura saturada, de gordura trans, de sódio, e de bebidas com baixo teor nutricional [Internet]. Diário Oficial [da] República Federativa do Brasil [acesso em 2010 jun 24]. Disponível em: <http://www.brasilsus.com. br/legislacoes/rdc/104537-24.html>.
Recebido em: 20/4/2011

Versão final em: 24/5/2012

Aprovado em: 5/6/2012 